\title{
A Rare Recurrence of an Immature Teratoma: A Case Report
}

\author{
Huang J, Zhang Z, Shi N, Yao S and Li D*
}

Hangzhou Obstetrics and Gynecology Hospital, Shangcheng District, Hangzhou City, Zhejiang Province, China

${ }^{*}$ Corresponding author: Li D, Hangzhou Obstetrics and Gynecology Hospital, No.369 KunPeng Road, Shangcheng District, Hangzhou City, Zhejiang Province, China, Tel: +8613588268798, E-mail: lidh2016@126. com

Citation: Huang J, Zhang Z, Shi N, Yao S, Li D (2018) A Rare Recurrence of an Immature Teratoma: A Case Report. J Gynecol Res 4(2): 202. doi: 10.15744/2454-3284.4.202

Received Date: December 2, 2017 Accepted Date: September 04, 2018 Published Date: September 06, 2018

\begin{abstract}
Objective: To state a rare case of immature teratomarecurrence, 14 years after the first surgery, which demonstrated as an immature teratoma, with nodules of glial implants.

Case report: A 26-year-old woman who was previously diagnosed as immature, right ovarian teratoma, was presented to Hangzhou obstetrics and gynecology hospital. The patient had previously undergone a laparotomy and right salpingo-oophorectomy with partial omentectomy 14-year before. Initial laboratory tests showed the serum markers: cancer antigen (CA)-125 was $44.5 \mathrm{IU} / \mathrm{ml}$ (increased) and CA199, AFP levels were normal. Sonographic examination revealed a complex mass, measuring $12.6 \mathrm{cmx} 10.5 \mathrm{~cm} \times 8.5 \mathrm{~cm}$, behind the uterus. Magnetic resonance imaging (MRI) findings were in favour of teratoma, originated from the right adnexa, occupying pouch of Douglas. The patient underwent exploratory laparotomy with a right intraligamentary teratoma cystectomy along with residual omentectomy,andrandom peritoneal biopsies. The pathology revealed mature teratoma, glioma, with no malignant components.

Conclusion: It is a rare recurrence of immature teratoma 14 years post-surgery. So, long-term follow-up is necessary for immature teratoma patients. And the case hints us that chemotherapy may transform an immature teratoma into a mature one. Andwe willgainan increased awareness of fertility sparing surgery in such young-aged patients in the future.
\end{abstract}

Keywords: Glioma; Implants; Immature teratoma; Mature teratoma; Recurrence

\section{Introduction}

Teratomas are the most common germ cell tumors. It can be categorized as either mature or immature. According to WHO, the definition of immature teratoma (IT) is a teratoma containing a variable amount of immature embryonal type, predominantly the neuroectodermal tissues [1]. IT is a relatively rare tumor that develops in the ovaries, and it accounts for $0.25-3 \%$ of tumors that develop in the ovaries [2]. We report a rare recurrence of immature teratoma in a 26-year-old woman 14 years post-surgery.

\section{Case presentation}

A 26-year-old female was admitted to our hospital with 21-day history of an enlarged abdominal mass. She has a significant past medical history- previously diagnosed as an immature, right ovarian teratoma and underwent a laparotomy and right salpingooophorectomy with partial omentectomy 14 years ago. The post-surgical pathology report confirmed that IT was Grade I-II, with implantation metastasis of omentum. So an official diagnosis was stage-III grade-II ovarian immature teratoma (Figure 1). Following surgery, adjuvant therapy had been administered, consisting of 6 cycles of BEP regime every 21 days (bleomycin in D2, D9 and D16, etoposide D1-5 and cisplatin D1-5, bleomycin $=15 \mathrm{U} / \mathrm{m}^{2}$, etoposide $=100 \mathrm{mg} / \mathrm{m}^{2}$ and cisplatin $\left.=20 \mathrm{mg} / \mathrm{m}^{2}\right)$. The patient had undertaken regular follow-up till three years before. No abnormal imagings or serum markers were found before the last follow-up. Recent serum markers showed that cancer antigen (CA)-125 was 44.5IU/ml (increased) and other tumor markers such as CA199, AFP levels were normal. Sonographic examination revealed a complex mass (Figure 2), measuring $12.6 \mathrm{cmx} 10.5 \mathrm{~cm}$ x8.5 cm, behind the uterus, no obvious blood flow signal was observed in Color Doppler Flow Imaging. Magnetic resonance imaging (MRI) findings were in favor of teratoma, originated from the right adnexa, occupying pouch of Douglas. The physical examination was normal, except for a large hard pelvic mass.

Based on the patient's medical history, recurrence of IT was suspected. Then, the patient underwent a sonographic examination of retroperitoneum and no abnormality was found. The contrast-enhanced upper abdominal CT scan revealed no significant focus. With consideration of the tumor size, the elevated serum markers, and her medical history, a diagnosis of an immature teratoma was 
considered. Therefore, the patient was scheduled for a laparotomy due to the suspicion of ovarian malignancy. Ascites was not obvious, normal saline was used to flush the abdominal cavity, and cytology examination was done. A giant tumor (Figure 3 ) about $12.0 \mathrm{~cm}^{\star} 10.0 \mathrm{~cm}^{\star} 8.0 \mathrm{~cm}$ in size was seen in the right broad ligament. Complete removal of the mass was successful. Solid nodules can be observed with a diameter of $0.5 \mathrm{~cm}$ to $2.0 \mathrm{~cm}$ on the surface of the residual omentum. So we did a residual omentectomy. Frozen pathology of the giant tumor was Solid teratoma, with no immature nerve components, and the nodules were reported as glioma. The cytology examination of the peritoneal fluid demonstrated no malignant cells. And no other abnormalities were found in pelvic and abdominal cavities. Subsequently, we tookrandom peritoneal biopsies, as the extensive sampling of all peritoneal implants are important [3]. The paraffin pathology reports were cystic and solid mature teratoma (Figure 4), with glial nodules in focal regions. The nodules were glioma (Figure 5), and biopsy specimens were normal tissues. Cytology examination demonstrated no malignant cells. The patient had no adjuvant treatment after the surgery and was scheduled for routine follow-up.

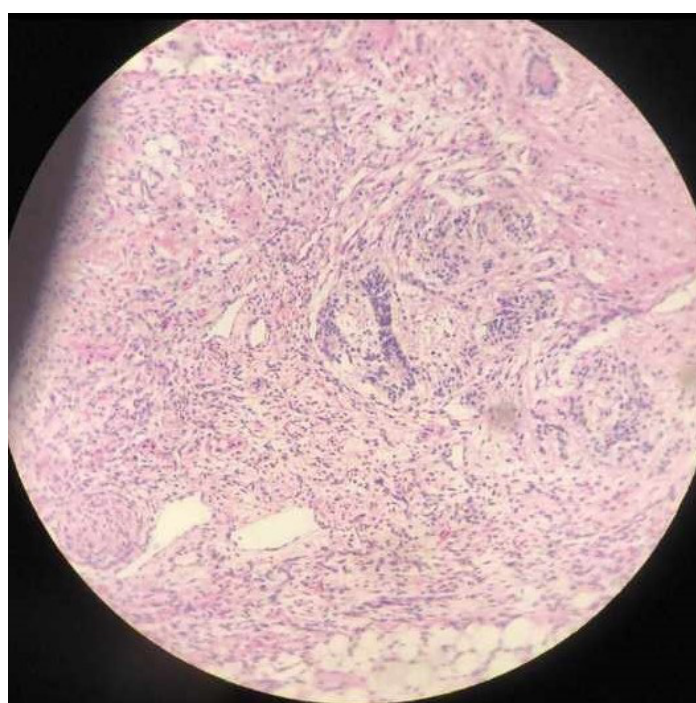

Figure 1: Microscopically, the post-surgical pathology report confirmed grade-II ovarian immature teratoma
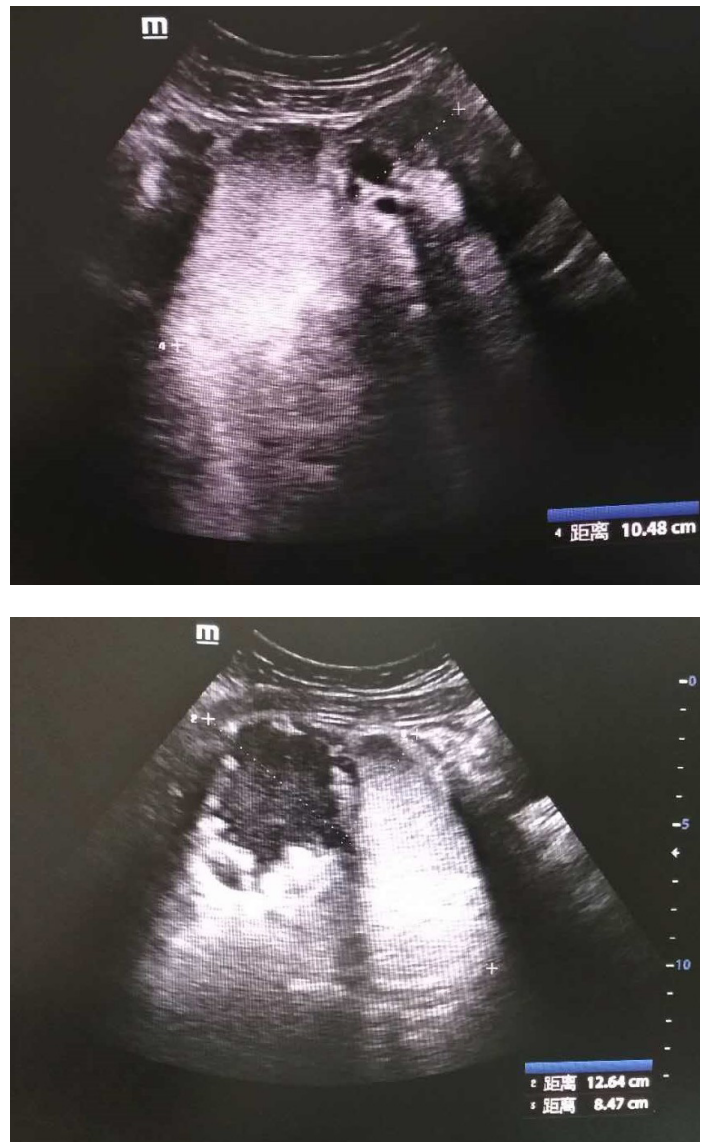

Figure 2: A complex mass measuring $12.6 \mathrm{cmx} 10.5 \mathrm{~cm}$ x 8.5 $\mathrm{cm}$ behind the uterus was observed in the sonographic examination 


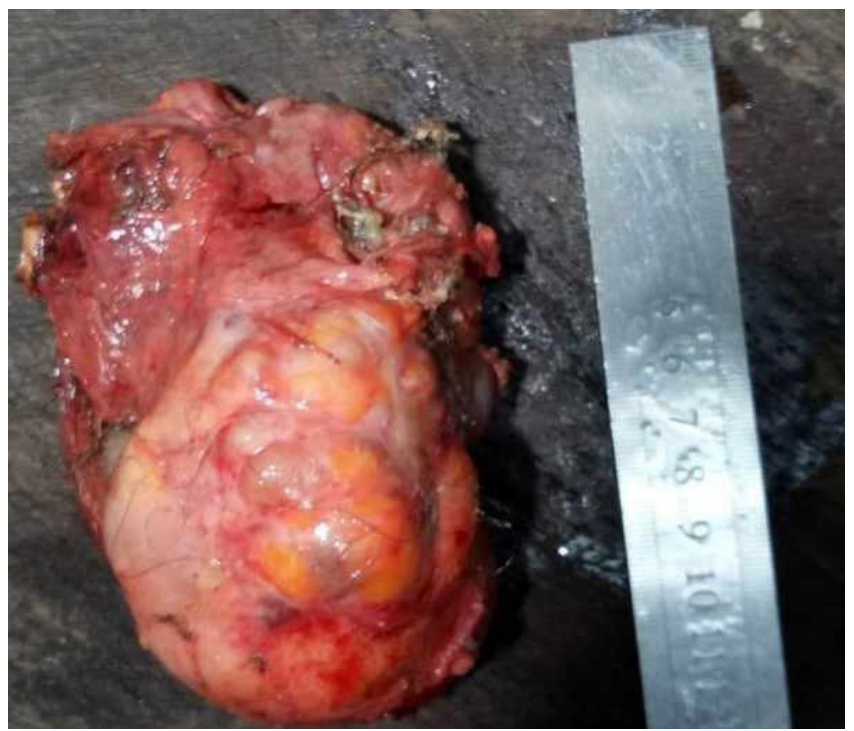

Figure 3: Complete removal of the giant tumorabout $12.0 \mathrm{~cm}^{\star} 10.0 \mathrm{~cm}^{\star} 8.0 \mathrm{~cm}$ in size from the right broad ligament
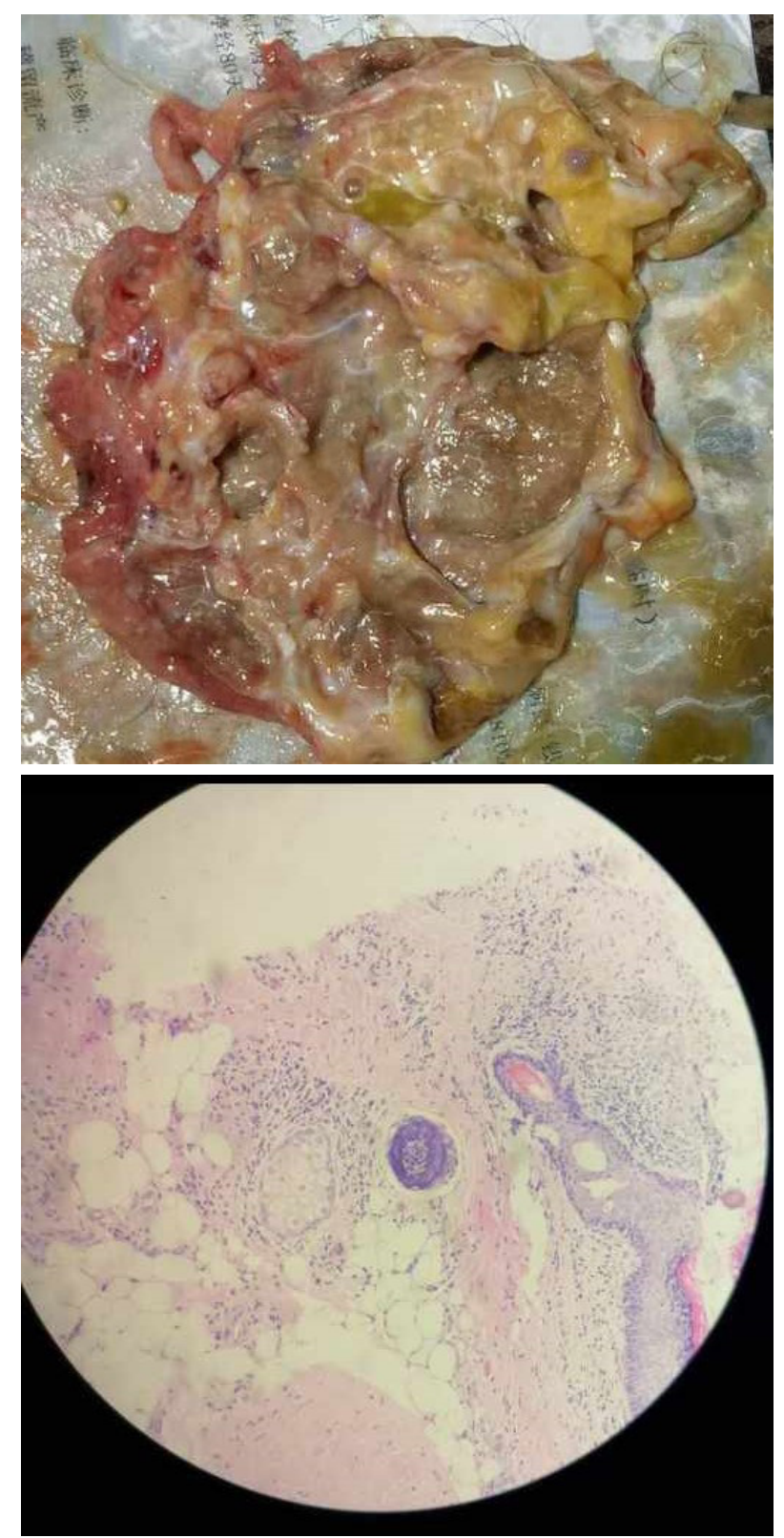

Figure 4: Macroscopically, the giant tumor was rich in sebaceous materials, and cartilage identified inside the mass. Microscopically, it demonstrated ascystic and solid mature teratoma 


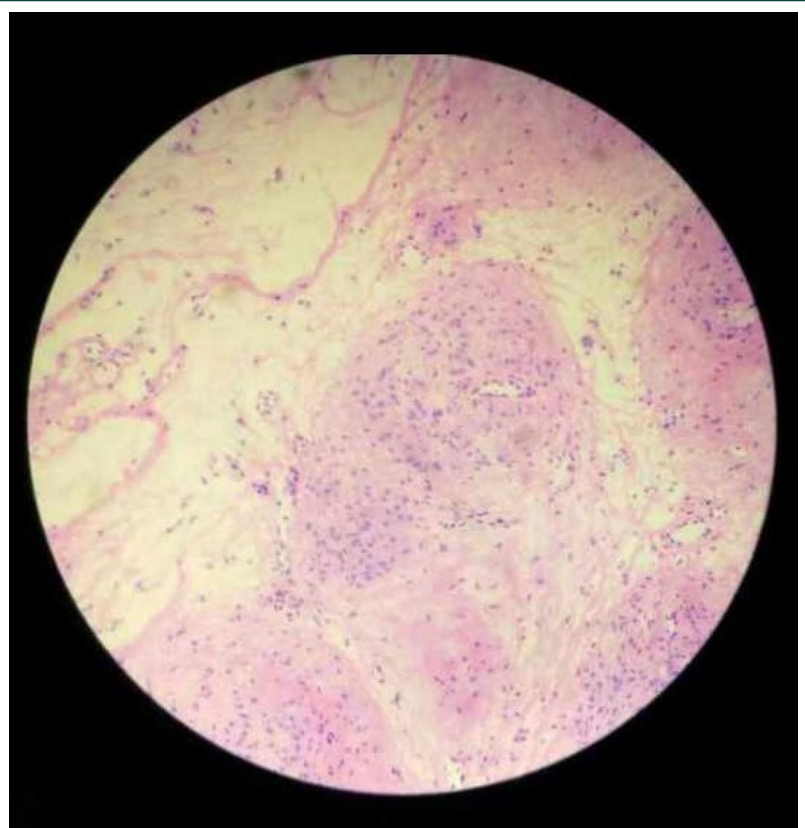

Figure 5: The nodules on the residual omentum were glioma, with no immature elements

\section{Discussion}

IT is a rare germ cell neoplasm accounting for $<1 \%$ of ovarian teratomas [4]. It typically presents in the first two decades of life. Local recurrence is usually within the first year of primary therapy initiation [5]. However, in this case, the rare recurrence is 14year after first treatment. Recurrent tumors should be excised to clarify diagnosis, and prevent possible malignant transformation [6]. So, the patient treated with an aggressive surgical therapy.

To our knowledge, Growing teratoma syndrome (GTS), is a disease characterized by presence of benign metastasis either during or after completion of chemotherapy, being chemo and radio-resistant, and in the presence of normal tumor markers. As it is reported so far, the latest development of GTS was 12 years after completion of therapy [7]. And in the case, the recurrence is 14 years later, with elevated tumor markers, so we don't recognize it as GTS, and it seems more likely as a recurrence. We inferred that the giant tumor in the right board ligament was the implantation of the initial IT.

The exact etiology of the disease is still unclear. As Andre F reported, chemotherapy may selectively inactivate the immature components and lengthen course of the disease, so benign components can grow further [8]. If so, we did not need to take radical operation. As preservation of fertility is a major concern for childbearing age patients, fertility sparing surgery are safe and recommended.

In this case, Gliomatosis peritonei (GP) was observed. GP is composed of mature glial tissue and can be located in the peritoneal cavity and omentum in patients with ovarian teratoma. It is formed by chemotherapeutic retro conversion of neuroectodermal elements [9]. It is in accordance with the omentum implantation in the time of first surgery.

The treatment for GP is complete surgical resection, which is also useful for identifying malignant lesions and for preventing malignancy transformation of the residual GP fragments. So we took a residual omentectomy. And it is reported that GP has a shorter recurrence-free survival [10]. Therefore, a longer follow-up period is necessary for the patient.

In summary, the recurrence has been late, in a very young woman. Maybe radical operation is not encouraged, especially in young age patients. The fertility preservation surgery is appropriate at diagnosis and that follow up should be longer in these cases.

\section{Conclusion}

It is a rare recurrence of immature teratoma 14 years post-surgery. So, long-term follow-up is necessary for immature teratoma patients. And the case hints us that chemotherapy may transform an immature teratoma into a mature one. And we will gain an increased awareness of fertility sparing surgery in such young-aged patients in the future.

\section{References}

1. Galateanu AG, Terzea DC, Carsote M, Poiana C (2013) Immature ovarian teratoma with unusual gliomatosis. J Ovarian Res 6: 28.

2. Saba L, Guerriero S, Sulcis R, Virgilio B, Melis G, et al. (2009) Mature and immature ovarian teratomas: CT, US and MR imaging characteristics. Eur J Radiol 72: 454-63.

3. Das CJ, Sharma R, Thulkar S, Mukhopadhyay S, Deka D, et al. (2005) Mature ovarian teratoma with gliomatosis peritonei-A case report. Indian J Cancer 42: $165-7$. 
4. Merard R, Ganesan R, Hirschowitz L (2015) Growing teratoma syndrome: A report of 2 cases and review of the literature Int J Gynecol Pathol 34: 465-72.

5. Barwad A, Dey P, Shivalingam J (2011) Metastatic of mature component in a treated case of immature teratoma diagnosed on fine needle aspiration cytology of the liver. Diagn Cytopathol 39: 711-3.

6. Amsalem H, Nadjari M, Prus D, Hiller N, Benshushan A (2004) Growing teratoma syndrome vs. chemotherapeutic retroconversion: Case report and review of the literature. Gynecol Oncol 92: 357-60.

7. Gorbatiy V, Spiess PE, Pisters LL (2004) The growing teratoma syndrome. Current review of the literature. Indian J Urol 25: 186-9.

8. Andre F, Fizazi K, Culine S, Droz J, Taupin P, et al. (2000) The growing teratoma syndrome: results of therapy and long-term follow-up of 33 patients. Eur J Cancer 36: 1389-94.

9. Mrabti H, El Ghissassi I, Sbitti Y, Amrani M, Hachi H, et al. (2011) Growing teratoma syndrome and peritoneal gliomatosis. Case Rep Med 2011: $10.1155 / 2011 / 123527$.

10. Yoon NR, Lee JW, Kim BG, Bae DS, Sohn I, et al. (2012) Gliomatosis peritonei is associated with frequent recurrence, but does not affect overall survival in patients with ovarian immature teratoma. Virchows Arch 461: 299-304.

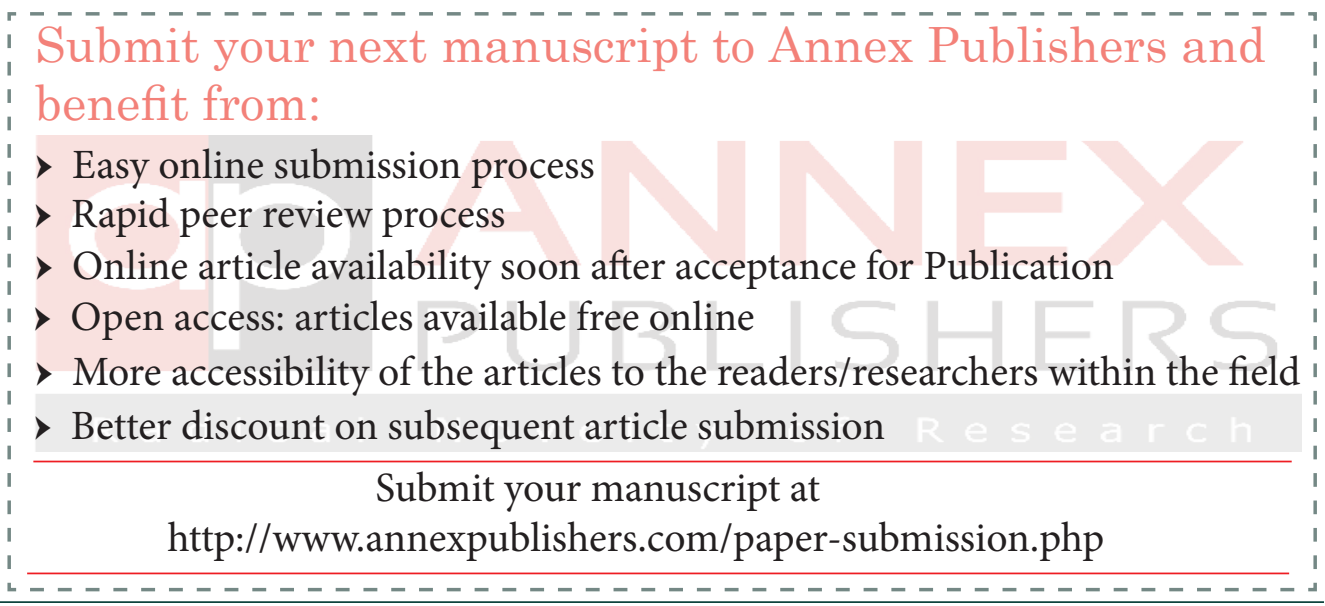

